\title{
Philosophiques
}

\section{Du sens littéral}

\section{Brendan S. Gillon}

Volume 33, numéro 1, printemps 2006

Philosophie et psychopathologie

URI : https://id.erudit.org/iderudit/012956ar

DOI : https://doi.org/10.7202/012956ar

Aller au sommaire du numéro

Éditeur(s)

Société de philosophie du Québec

ISSN

0316-2923 (imprimé)

1492-1391 (numérique)

Découvrir la revue

Citer ce document

Gillon, B. S. (2006). Du sens littéral. Philosophiques, 33(1), 237-248.

https://doi.org/10.7202/012956ar d'utilisation que vous pouvez consulter en ligne.

https://apropos.erudit.org/fr/usagers/politique-dutilisation/ 


\title{
Du sens littéral'
}

\author{
BRENDAN S. GILLON \\ Université McGill
}

Quelques suppositions semblent incontournables dans l'étude du langage. La première est que toute expression complexe d'une langue doit être analysée dans ses plus petits éléments, c'est à dire ceux que les linguistes appellent des morphèmes. La deuxième est que le sens d'une expression complexe est déterminé par le sens des expressions minimales qui la constituent et par la structure syntaxique qui forme, à partir d'elles, l'expression complexe ellemême. La troisième est que chaque expression minimale a un sens invariant. Ces trois suppositions ont guidé toutes les études sérieuses du langage depuis leur début, il y a plus de deux mille cinq cents ans, quand le grand grammairien du sanscrit Pānịni a rédigé la première grammaire générative.

Un aspect du langage naturel que l'on reconnaît depuis très longtemps est le fait que l'interprétation d'une expression peut être relative à la situation dans laquelle on l'énonce. Toutefois, le traitement formel de la sémantique des expressions clairement identifiées comme telles n'est apparu que pendant la seconde moitié du vingtième siècle.

Deux sortes d'expressions ont été clairement identifiées. Une des sortes est appelée : endophore, et l'autre : exophore. Pour comprendre ce que veut dire endophore, considérons les deux phrases suivantes :

1.1 Deux est un nombre pair.

1.2 Il est un nombre pair.

Non seulement les deux phrases sont grammaticalement complètes, mais elles ont la même structure syntaxique. Bien que la première soit vraie, nous ne pouvons pas dire, à défaut de tout contexte, si la seconde est vraie ou fausse.

Ensuite, considérons deux autres phrases, toutes deux vraies :

2.1 Deux est un nombre premier.

2.2 Trois est un nombre premier.

Finalement, adjoignons la phrase 1.2 à chacune des phrases en 2, ce qui donne les deux phrases ci-dessous :

3.1 Deux est un nombre premier, et il est pair ;

3.2 Trois est un nombre premier, et il est pair.

Même sans aucune connaissance de la situation de leur énonciation, on peut juger la première, vraie, et la seconde, fausse.

1. Je tiens à remercier Michel Paradis et Paul Pupier pour toutes les améliorations qu'ils ont apportées à mon français. Je remercie aussi Steven Davis pour ses commentaires. 
Évidemment, l'interprétation de la phrase dans 1.2 peut dépendre du texte qui l'entoure, ce que j'appelle son co-texte. Je désigne par endophore toute expression qui en exige une autre dans le co-texte pour que son interprétation soit complète. J'appelle antécédent le constituant minimal, dans le cotexte, qui détermine l'interprétation de l'endophore. Ainsi, dans la première phrase coordonnée ci-dessus, l'antécédent du pronom personnel de la troisième personne du singulier est deux, et, dans la deuxième, l'antécédent est trois. (Cette relation est signalée par le soulignage de l'endophore et de son antécédent.)

Considérons, à son tour, ce que j'appelle une expression exophore. Le contexte de son énonciation doit être connu pour que son interprétation soit complète. Cette expression est aussi appelée déictique par les linguistes, et indicateur (indexical) par les philosophes analytiques anglophones. La première phrase suivante en est un exemple.

4.1 Marco Polo est mort ici.

4.2 Marco Polo est mort à Venise.

La deuxième phrase est vraie, comme n'importe quelle personne connaissant suffisamment la vie de Marco Polo peut le constater, tandis que même un expert de la vie de Marco Polo ne peut pas déterminer la vérité de la première phrase sans une connaissance de la situation dans laquelle la phrase a été énoncée. J'appelle la situation physique dans laquelle une phrase est énoncée sa scène.

Pour savoir si une expression est exophorique, on peut toujours la mettre à l'épreuve. On choisit une phrase contenant l'expression dont on veut déterminer si elle est ou non exophorique, et qui est assujettie à un jugement de vérité ; puis, en gardant les mêmes conditions de vérité, on change la situation de son énonciation et on constate si le jugement de la vérité de la phrase en est ou non changé. De la même façon, on peut constater si une expression est ou non endophorique en gardant les mêmes conditions de vérité et en changeant le co-texte. Comme Quine (1960, $\$ 27$, pp. 131-132) l'a souligné, on ne doit pas confondre, en jugeant de la valeur de vérité d'une phrase, le changement qui résulte du fait qu'elle contient des expressions exophoriques ou, d'ailleurs, endophoriques - avec le changement qui résulte du fait qu'elle contient des expressions ambiguës. L'ambiguïté engendre l'assujettissement des phrases à un jugement de vérité, sans que rien ne soit changé dans les conditions de vérité, dans la situation de l'énonciation ou dans le co-texte. (Voir Gillon, 2004.)

Même si quelques expressions ont un emploi endophorique et pas d'emploi exophorique - par exemple, le pronom réfléchi se - et même si d'autres ont un emploi exophorique et pas d'emploi endophorique - par exemple, le pronom personnel de la première personne du singulier et l'adverbe maintenant -, plusieurs expressions ont les deux emplois. C'est le cas des pronoms personnels de la troisième personne. 
L'identification de ces deux sortes d'expressions est le résultat des travaux des linguistes aussi bien que ceux des philosophes. Cependant, leur traitement sémantique est le fruit du travail des philosophes. L'analyse de la sémantique des expressions exophoriques a été entamée par Bar-Hillel (1954) et perfectionnée par Kaplan (1977), Lewis (1979) et Stalnaker (1970), et celle de la sémantique des expressions endophoriques par Geach (1962) et Quine (1960). Disons que le contexte grammatical est l'ensemble des aspects de la situation d'énonciation d'une phrase exigés par les mots qui forment celle-ci pour qu'elle acquière des conditions de vérité ${ }^{2}$. La scène et le co-texte sont donc contenus dans ce contexte. Mais ce fait nous conduit à la question suivante : sont-ils les seuls aspects du contexte grammatical ? Autrement dit, les conditions de vérité d'une phrase grammaticale dépendent-elles de la présence exclusive des endophores et des exophores dans le contexte ? Plusieurs Sperber et Wilson (1986), Bach (1999), Carston (2002), Recanati, Levinson (2004), et d'autres encore - ne le pensent pas. Une bonne partie des données en faveur de l'hypothèse que le contexte doit comprendre plus que le co-texte et la scène est présentée dans le livre de François Recanati intitulé Literal Meaning, publié tout récemment à la Cambridge University Press.

Dans ce qui suit, je montrerai que plusieurs de ces données ne justifient pas l'élargissement du concept de contexte grammatical au-delà de la scène et du co-texte. Toutes les données que je discuterai exemplifient la même chose, à savoir qu'on peut arriver à communiquer une circonstance par une phrase, même si cette circonstance ne fait pas partie de ses conditions de vérité, ou même si la phrase n'a pas de conditions de vérité. Je distingue donc entre les conditions de vérité d'une phrase (dites aussi les circonstances d'évaluation d'une phrase), d'une part, et la circonstance que le locuteur tente d'exprimer par sa phrase ou que l'allocutaire pense que son locuteur tente d'exprimer, d'autre part. Autrement dit, je distingue entre le sens d'une phrase déterminé par le sens invariant de ses mots et de sa structure syntaxique, d'un côté, et le sens que le locuteur de la phrase tente d'exprimer par la phrase ou celui que l'allocutaire attribue à la phrase énoncée par le locuteur, de l'autre.

Les exemples les plus frappants de l'écart entre le sens d'une phrase énoncée dans une situation et le sens visé par le locuteur, ou le sens qui lui est attribué par l'allocutaire, sont ceux où la langue d'expression n'est ni la langue maternelle du locuteur ni celle de l'allocutaire.

Même dans de tels cas, l'allocutaire dont la langue maternelle n'est pas celle de la phrase énoncée n'a aucune difficulté à comprendre ce que le locuteur veut communiquer, bien que la phrase contienne une erreur de genre ou d'accord, par exemple. Nous n'avons qu'à penser aux phrases prononcées par les personnages dont la langue maternelle n'est pas le français dans la bande dessinée Tintin.

2. Pour simplifier la discussion, je me limite aux phrases déclaratives susceptibles de posséder des conditions de vérité. 
De la même façon, les adultes comprennent facilement ce que leurs enfants veulent leur communiquer, bien que les phrases énoncées par ceux-ci contiennent des erreurs grammaticales. Sans doute, il y a des malentendus, comme l'illustre bien un dessin humoristique. Dans ce dessin, une mère, en train de préparer un repas dans la cuisine, entend son enfant lui dire qu'il vient de scier en deux le pied d'une chaise dans la pièce à côté : Mommy, I just sawed the chair ("Maman, je viens de scier la chaise en deux "), et sa mère le corrige en disant : No dear, you saw the chair ("Non chéri, tu as vu la chaise »).

Même les adultes énoncent des phrases dont le sens ne correspond pas à ce qu'ils veulent dire. On connaît, bien sûr, le fameux révérend William A. Spooner (1844-1930), ancien chef du New College de l'Université Oxford, réputé pour ses erreurs linguistiques humoristiques non intentionnelles. Par exemple, on lui attribue l'énonciation de la première phrase suivante, par laquelle il voulait exprimer le sens de la deuxième phrase.

5.1 Work is the curse of the drinking class.

Le travail est le fléau de la classe qui boit.

5.2 Drink is the curse of the working class.

La boisson est le fléau de la classe ouvrière.

De pareils cas abondent partout, à l'écrit et à l'oral. Quelqu'un m'a dit une fois la phrase énoncée dans 6.1, en voulant dire la phrase énoncée dans 6.2.

6.1 I did not hear the computer until I turned it off. Je n'ai entendu l'ordinateur qu'après l'avoir éteint.

6.2 I had not noticed the noise the computer had been making until I turned it off.

Je n'ai remarqué le bruit fait par l'ordinateur qu'après avoir éteint celui-ci.

Un passager dans ma voiture m'a dit, pendant que je conduisais, la phrase énoncée dans 7.1, en voulant dire celle énoncée dans 7.2.

7.1 The traffic is slippery.

La circulation est glissante.

7.2 The road is slippery.

La chaussée est glissante.

Si ces exemples anecdotiques ne nous ont pas persuadés, considérons alors des exemples publiés. La phrase suivante se trouvait dans un article de U.S.A. Today (14 nov. 1996, p. 1) :

8. John Dalton had refused to rent to black customers for ten years. Pendant dix ans, John Dalton avait refusé de louer aux clients noirs.

Comme l'article l'explique, ce que John Dalton avait refusé de faire pendant dix ans, c'était de louer aux Noirs. Mais les personnes à qui quelqu'un refuse de louer sont-elles ses clients? 
Voici une phrase tirée de l'International Herald Tribune (Sat.-Sun., July 2-3, 2005, p. 20) :

9. A thirteen-year old girl was killed after being bitten by a shark. Une fille de treize ans a été tuée après avoir été mordue par un requin.

Mais rien dans l'article ne nous laisse entendre que la malheureuse fille a survécu à l'attaque d'un requin, mais a été tuée plus tard.

Il y a peu de chances que la plupart des lecteurs aient compris ces deux phrases autrement que de la façon suivante :

10.1 John Dalton avait refusé de louer aux Noirs pendant dix ans.

10.2 Une fille de treize ans est morte après qu'un requin l'a mordue.

Il n'est pas rare qu'un locuteur et son allocutaire s'entendent parfaitement, c'est-à-dire que le sens attribué par l'allocutaire à une phrase soit précisément le sens visé par le locuteur, bien que le sens visé et le sens attribué ne soient pas le sens de la phrase énoncée. Voici un exemple dont j'ai été témoin.

11.1 A : Peux-tu voir le tableau noir ?

11.2 B : Oui.

Bien sûr, on peut facilement imaginer cet échange entre un conférencier et un membre de son auditoire. En fait, cet échange a eu lieu entre une mère et son fils, les deux sachant que le fils pouvait avoir besoin de lunettes. Pourtant, à cette occasion, aucune allusion à cette possibilité n'a été faite. Par la question énoncée dans 11.1, dont le contresens est identique à celui de la question 6 , la mère visait celle énoncée dans 12.

12. Peux-tu voir ce qui est écrit sur le tableau noir ?

Et ce que le fils voulait faire comprendre à sa mère en répondant oui, c'est qu'il pouvait lire ce qui était écrit sur le tableau noir.

Considérons un cas bien discuté dans la philosophie du langage. Keith Donnellan (1966) a observé qu'une phrase contenant un syntagme nominal défini peut communiquer la circonstance à laquelle le locuteur faisait allusion en énonçant la phrase, malgré le fait que rien dans les circonstances d'évaluation ne satisfait le contenu descriptif du syntagme nominal. Imaginez une réception ; une seule personne à cette réception est en train de boire une boisson gazeuse dans un verre de champagne. Imaginez aussi que deux autres personnes la regardent et que l'une d'elles dise à l'autre la phrase suivante :

13. La personne qui boit du champagne est un philosophe.

La phrase communiquera que la personne regardée par les deux autres est un philosophe, et ce, même si le verre ne contient pas de champagne, mais, disons, du Perrier. Si la personne que les deux autres regardent boit du Perrier, la phrase n'est pas vraie. Pourtant, dans la situation envisagée, le locuteur communique 
la circonstance qu'il veut communiquer, c'est-à-dire que la personne qu'il regarde est un philosophe, bien que la phrase énoncée ne soit pas vraie.

Les syntagmes nominaux déterminés par l'article défini ne sont pas les seuls dont le sens échoue à identifier le sens visé par le locuteur. Cela peut arriver aux syntagmes nominaux dont le déterminant est une exophore. Restons à la réception à laquelle assistent Charles et son colocataire, Marc. Charles a amorcé une conversation avec, disons, Alice. Après quelques minutes, Charles se rend compte qu'il n'a pas présenté Marc à Alice. Pendant ce temps, à l'insu de Charles, Marc s'est éloigné. Charles, sans regarder l'endroit que Marc a occupé il y a quelques instants, dit :

14. Voici mon colocataire, Marc.

Dans cette scène, l'exophore voici n'a pas la valeur voulue par son locuteur. Malgré cela, la phrase peut communiquer exactement ce que voulait son énonceur.

Ce n'est pas toujours l'erreur qui mène à un écart entre le sens d'une phrase et ce que le locuteur veut communiquer ou ce que l'allocutaire comprend. Parfois, le locuteur choisit, exprès, une phrase dont le sens est différent du sens visé, et ce, pour que son allocutaire reconnaisse l'écart et, par ce moyen, sache ce qu'il voulait communiquer. C'est-à-dire que le locuteur énonce une phrase sarcastiquement. En voici un exemple.

Supposons qu'Alain et Bertrand, qui ont une connaissance de la géographie de l'Europe, se parlent. Supposons aussi qu'Alain a une assez faible connaissance de la géographie du Moyen Orient. La première phrase est énoncée dans 15. Bertrand emploie la deuxième phrase pour corriger ce qu'Alain a exprimé par la première.

\section{Alain : Téhéran est en Turquie.}

Bertrand : Et Londres est en France.

Pour que Bertrand réussisse à communiquer à Alain que ce qu'il a dit est faux, il est nécessaire qu'Alain reconnaisse que la phrase de Bertrand est fausse et qu'il reconnaisse aussi que Bertrand le sait également. Donc, Alain doit comprendre le sens de la phrase de Bertrand, reconnaître la fausseté de celle-ci et conclure que ce qu'il a dit est faux.

Muni de la distinction entre le sens d'une expression, d'un côté, et le sens que le locuteur vise à exprimer en l'énonçant ou le sens que son allocutaire lui attribue en l'entendant, réfléchissons sur plusieurs données citées par Recanati. Ce que ces données montrent, c'est que les locuteurs sont indulgents envers le laxisme sémantique de leurs partenaires dans leurs conversations.

Commençons par une observation qui a contrarié David Lewis. Dans son célèbre article, "Scorekeeping in a language game » (1979), il a introduit le concept d'accommodement. Selon Lewis, l'accommodement est une sorte d'ajustement fait par les interlocuteurs pour réconcilier le sens d'une expression avec le sens qu'ils voulaient se communiquer dans le but de faciliter leur 
communication. L'auteur donne l'exemple de l'accommodement fait par les interlocuteurs pour trouver des normes de précision convenables au bon déroulement de la conversation portant sur l'emploi des adjectifs qualificatifs. Pour illustrer son idée, Lewis reprend un exemple de John Austin. On peut dire que :

\section{6. la France est hexagonale,}

bien qu'elle n'ait pas vraiment la forme d'un hexagone. La raison pour laquelle un allocutaire accepte une telle phrase comme vraie, selon Lewis, c'est qu'il s'accommode aux normes évidemment moins strictes de son locuteur dans le but de ne pas entraver la bonne progression de la conversation.

Une conséquence de son hypothèse, nous dit Lewis, c'est qu'un allocutaire a également la possibilité d'adopter une norme plus stricte ou moins stricte. Pourtant, comme il le note, en réalité, il n'y a pas de symétrie : adopter des normes plus strictes passe mieux que d'adopter des normes moins strictes, et cela même quand le resserrement de normes empêche la conversation.

Ce que Lewis n'a pas observé, c'est que cette asymétrie ne se borne pas aux adjectifs qualificatifs. La même asymétrie se manifeste à travers les adverbes comme maintenant et $i c i$, et les syntagmes nominaux quantificationnels.

Prenons la remarque de Recanati (2004, pp. 57-58) :

La valeur sémantique [de now (« maintenant ») et de here (« ici »)] est respectivement le temps ou le lieu du contexte. Mais, quel temps et quel lieu sont considérés comme le contexte ? Quelle partie de temps ou de lieu est incluse ? Cela dépend de l'intention du locuteur, et donc, encore une fois, du contexte au sens large. Comme tout le monde le sait, here [« ici »] peut référer à cette chambre, à ce bâtiment, à cette ville, à ce pays, etc., et la même sous-détermination affecte now [" maintenant»].

Ce qui est en jeu dans l'emploi de ces deux mots n'est pas du tout la sousdétermination, mais plutôt le laxisme des normes. Et, comme Lewis l'a remarqué dans le cas des adjectifs qualificatifs, il y a une asymétrie. On peut toujours refuser l'emploi sémantiquement laxiste des mots comme «maintenant » (now) et «ici» (here).

Imaginons deux personnes dans un bureau à Montréal. Une des deux reçoit un appel téléphonique de Guillaume, qui est venu de New York et s'est installé dans sa chambre d'hôtel. La personne qui a reçu l'appel dit à l'autre :

17. Guillaume est ici.

L'autre personne peut très bien nier la vérité de la phrase dans 17 : après tout, Guillaume n'est pas dans le bureau ; il est dans sa chambre d'hôtel. La première personne a énoncé une phrase strictement fausse, mais elle a réussi néanmoins à communiquer ce qu'elle voulait, à savoir, selon l'exemple, que Guillaume est à Montréal. 
Je laisse au lecteur le soin de formuler un exemple pareil pour l'adverbe « maintenant» $($ now $)$.

Le même phénomène se manifeste dans l'emploi des syntagmes nominaux quantificationnels, tellement discuté aujourd'hui sous le nom de " restriction du domaine de quantificateurs " (quantifier domain restriction). On lit souvent que ce que voulait dire un locuteur en énonçant une phrase comme :

18. Tout le monde est venu à la réception,

n'est pas strictement que tout le monde est venu, mais que tous, dans un domaine restreint, disons tous les amis de l'énonciateur de la phrase dans 18, sont venus. Cependant, le domaine du discours ne peut pas fournir une contrainte supplémentaire au syntagme nominal quantificationnel pour qu'une phrase exprime ce que le locuteur tente d'exprimer en l'énonçant. La phrase dans 19.1 communique le sens de la phrase dans 19.2.

19.1 Jean déteste tout le monde.

19.2 Jean déteste tout le monde sauf lui-même.

Puisque le syntagme nominal Jean est dans la phrase, sa référence doit être dans le domaine du discours. Et puisque Jean est une personne, il doit être dans le domaine du syntagme nominal quantificationnel tout le monde. La situation est tout à fait la même pour la phrase énoncée dans 20.1, qui communique le sens de celle énoncée dans 20.2.

$20.1 \quad$ Tout est sur la table.

20.2 Tout, sauf la table, est sur la table.

Par contre, dans 19 comme dans 20, les phrases ne sont pas synonymes. La conséquence logique de la première phrase dans 19 est la première phrase dans 21 ; mais la conséquence logique de la deuxième phrase dans 19 n'est pas la première phrase dans 21 .

$21.1 \quad$ Jean se déteste.

21.2 La table est sur elle-même.

De la même façon, la conséquence logique de la première phrase dans 20 n'est pas la deuxième phrase dans 21 ; mais la conséquence logique de la deuxième phrase dans 20 n'est pas la deuxième phrase dans 21. Le problème de la restriction du domaine du quantificateur n'est pas unique aux syntagmes nominaux quantificationnels. Le problème des restrictions existe aussi pour certains adjectifs. Considérons la phrase suivante, que j’ai lue dans un texte sur la philosophie.

22. Would someone row away from a sinking ship in an empty lifeboat? Qui s'éloignerait à la rame d'un navire qui coule dans un canot de sauvetage vide? 
En réfléchissant sur cette question, on arrive à s'en poser une autre, à savoir :

23. If the lifeboat is empty, who is rowing?

Si le canot de sauvetage est vide, qui rame ? Évidemment, ce que l'on voulait demander, c'est :

24. Would someone row away from a sinking ship alone in a lifeboat? Qui s'éloignerait à la rame d'un navire qui coule, tout seul dans un canot de sauvetage?

Le même phénomène se manifeste de nouveau dans les cas de prétendues "descriptions définies incomplètes" (incomplete definite descriptions). Ces cas sont, en quelque sorte, l'inverse des cas de Donnellan mentionnés plus haut, où le syntagme nominal décrit mal l'objet visé par le locuteur. Dans le cas des descriptions définies incomplètes, le syntagme nominal décrit l'objet visé correctement, mais ne le décrit pas assez pour spécifier uniquement l'objet visé. Voici un exemple de James McCawley :

24. Le chien a mordu un autre chien. The dog bit another dog.

Les deux syntagmes nominaux exigent que le domaine de discours contienne au moins deux chiens. Par contre, le premier syntagme nominal, une description définie, exige qu'il n'y ait qu'un chien. Néanmoins, le locuteur d'une telle phrase peut très bien communiquer la circonstance comme il le désire en énonçant la phrase dans 24.

Dans les cas précédents, les phrases des données étaient soit mal formées soit bien formées, mais littéralement fausses. Pourtant, de telles phrases peuvent réussir - et normalement elles réussissent — à communiquer la circonstance que leur locuteur désire exprimer en les énonçant. Tournons-nous vers les phrases bien formées et littéralement vraies, qui peuvent communiquer une circonstance mais pas nécessairement celle visée par le locuteur.

Commençons avec un exemple tiré des Histoires d'Hérodote. Cet ancien Grec relate que le roi de Lydie, Crésus, envisageant de faire la guerre contre Cyrus le Grand, roi des Perses, envoya un messager au temple d'Apollon, à Delphes, pour demander au dieu s'il devait faire la guerre contre Cyrus. La Pythie, la femme qui servait à transmettre la réponse du dieu, dit que « si Crésus attaquait les Perses, il détruirait un grand empire » (chap. 53). Crésus, ravi de la réponse, attaqua, mais Cyrus le vainquit et le fit prisonnier. Crésus demanda alors à celui-ci de lui permettre d'envoyer un messager au temple d'Apollon pour demander si le dieu " n'avait pas honte de l'avoir encouragé à lancer une guerre contre les Perses, qui devait porter de tels fruits, en lui disant qu'il était destiné à détruire l'empire de Cyrus " (chap. 90). La Pythie répondit que "s'il avait été sage, il aurait envoyé un messager une deuxième fois pour demander à quel empire le dieu faisait allusion, celui de Cyrus ou le sien » (chap. 91). 
Bien que l'empire perse et l'empire lydien aient été dans le domaine du discours et dans le domaine du syntagme nominal un grand empire, Crésus a interprété la réponse de la Pythie plus étroitement que ne le permettait le sens de la phrase. Autrement dit, la réponse à la première question posée par Crésus était sous-spécifiée par rapport à ce que Crésus voulait savoir d'Apollon, comme le confirme la réponse de la Pythie à la deuxième question que Crésus avait posée.

Normalement, la sous-spécification ne nous donne pas d'ennuis. Prenons l'exemple :

\section{Avez-vous mangé ?}

La question ne spécifie pas quand. Normalement, on comprend qu'elle est posée par rapport au jour pendant lequel on la pose, comme dans la question suivante :

26. Avez-vous mangé le petit déjeuner ?

Mais, normalement, aucune limite de temps n'est comprise dans la question posée dans 27 :

27. Avez-vous mangé du caviar ?

Dans ce cas, la question se pose par rapport à la vie de la personne interrogée. Pourtant, il n'y a aucune raison de penser que cette différence résulte de quelque élément de la grammaire ou du lexique. Au contraire, tout indique que le rapport au temps est sous-spécifié.

La sous-spécification n'est pas le caractère exclusif des syntagmes nominaux indéfinis et du temps. Même les exophores, comme les pronoms démonstratifs, permettent la sous-spécification, comme en témoigne un exemple de Ludwig Wittgenstein, adapté ci-dessous. Imaginez quelqu'un qui montre du doigt une bouteille de vin et dit :

28. Ceci est importé.

Le locuteur peut vouloir communiquer, en énonçant cette phrase, que le vin dans la bouteille est importé, ou il peut vouloir communiquer que le verre de la bouteille est importé. La phrase, même assortie du geste, est sous-spécifiée par rapport à ces deux possibilités. Les deux phrases suivantes, dans la même situation d'énonciation, ne sont pas sous-spécifiées de cette façon.

29.1 Ce vin est importé.

29.2 Ce verre est importé.

C'est encore la sous-spécification qui est en jeu dans les exemples cités par Recanati, comme les phrases suivantes.

30.1 Mary took out her key and opened the door.

Marie a sorti sa clef et a ouvert la porte. 


\subsection{The policeman stopped the car.}

Le policier a arrêté la voiture.

Il n'y a rien dans le sens de la première phrase qui exige que Marie se soit servie de sa clef pour ouvrir la porte; et il n'y a rien dans le sens de la deuxième phrase qui détermine comment le policier a arrêté la voiture. C'est absolument compatible avec le sens de la deuxième phrase, par exemple, que le policier était conducteur et a arrêté la voiture en appuyant sur le frein ou qu'il était en train de contrôler la circulation et a signalé au conducteur de la voiture de s'arrêter.

Dans chaque cas examiné, nous avons vu qu'une phrase peut réussir à communiquer une circonstance qui n'est pas parmi les conditions de vérité déterminées par son sens. Aucun de ces cas ne donne à penser que la circonstance fait partie des conditions de vérité fixées par un deuxième sens déterminé par la grammaire et le lexique. Donc, les cas examinés ne donnent pas à penser qu'on doit élargir le concept de contexte grammatical pour inclure quoi que ce soit au-delà de la scène et du co-texte. Cela dit, nous ne pouvons pas exclure la possibilité que d'autres données puissent nous obliger à élargir le concept de contexte grammatical.

Recanati cite plusieurs phénomènes contrariants. Prenons, par exemple, le problème de l'interprétation de la préposition « de » (of) dans un syntagme prépositionnel. Comme on le sait depuis le temps de Pānịni, la contribution sémantique de la préposition est très variée. Si le nom qui a pour complément un syntagme prépositionnel est un nom relationnel non dérivé comme "frère ", ou un nom dérivé d'un verbe comme "mort ", l'interprétation de la préposition est déterminée par la relation comprise dans le sens du nom dont le complément est un tel syntagme. Donc, la préposition « de » dans le syntagme nominal " un frère de Jean » signale que Jean est un des relata de la relation de frère, tout comme " de » dans le syntagme nominal « la mort de Jean » signale à qui la mort est attribuée. Mais, l'interprétation devient moins claire dans les cas où le nom dont le syntagme prépositionnel est le complément n'est ni dérivé d'un verbe ni relationnel. Par exemple, dans l'expression " voiture de Jean ", s'agit-il de la voiture qui appartient à Jean, de la voiture que Jean a louée, ou de la voiture que Jean a empruntée à son frère ? Je ne peux entrer ici dans les détails de ce problème. Il suffit de dire qu'il n'existe pas, à ce jour, d'analyse reconnue par les linguistes comme satisfaisante. Recanati mentionne encore, entre autres problèmes, ceux du flou et de la polysémie. Mais sans une analyse convaincante de ces phénomènes, on ne peut en tirer quelque conclusion que ce soit, et donc rien sur la nature du concept de contexte.

\section{Bibliographie}

Bach, Kent. "The Myth of Conversational Implicature ", Linguistics and Philosophy, vol. 22, 1999, pp. 327-366.

Bar-Hillel, Y. « Indexical Expressions », Mind, vol. 63, 1999, pp. 689-690. 
Carston, Robyn. Thoughts and Utterances : the Pragmatics of Explicit Communication, Oxford, England, Blackwell, 2002.

Davis, Steven et Brendan Gillon (dir.). Semantics : A Reader, Oxford, England, Oxford University Press, 2004.

Donnellan, Keith. "Reference and Definite Descriptions ", The Philosophical Review, vol. 75, n. 3, 1966, pp. 281-304.

Geach, Peter. Reference and Generality, Ithaca, New York, Cornell University Press, 1962.

Gillon, Brendan S. «Ambiguity, Indeterminacy, Deixis and Vagueness : Evidence and Theory ». Dans Davis et Gillon, 2004, pp. 157-187.

Kaplan, David. 1977. "Demonstratives ». Manuscrit. Réimprimé dans Davis et Gillon, 2004, pp. 749-799.

Levinson, Stephen C. Presumptive Meanings : the Theory of Generalized Conversational Implicatures, Cambridge, Massachussetts, The MIT Press, 2000.

Lewis, David. 1970. "Scorekeeping in a Language Game », Journal of Philosophical Logic, vol. 8, pp. 339-359. Réimprimé dans Davis et Gillon, 2004, pp. 803-816.

Quine, Willard Van Orman. 1960. Word and Object, Cambridge, Massachusetts, The MIT Press.

Recanati, François. Literal Meaning, Cambridge, England, Cambridge University Press, 2004.

Sperber, Daniel et Deidre Wilson. 1986. Relevance : Communication and Cognition, Oxford, England, Blackwell, $2^{\mathrm{e}}$ édition, 1995.

Stalnaker, Robert. "Pragmatics », Synthèse, vol. 22, 1970, pp. 272-289. 